\title{
Guidelines on the use of liver biopsy in clinical practice
}

\author{
A Grant, J Neuberger
}

\subsection{Introduction}

Erlich is credited with the first liver aspiration in 1883 and subsequently the first percutaneous liver biopsy for diagnostic purposes was reported in $1923 .{ }^{1}$ The technique has been modified since then, and over the past 50 years it has become a central investigation of hepatic disease. The low mortality $(0.01-0.17 \%)$ and the relatively low morbidity of this procedure have meant that liver biopsy has become widely used. $^{2}$

Advances in medical technology and especially in imaging, together with advances in drug therapy have greatly influenced the diagnosis and management of hepatic disease and as a consequence the indications for liver biopsy are changing. In 1991 the British Society of Gastroenterology (BSG), together with the Royal College of Physicians of London, undertook a nationwide audit of percutaneous liver biopsy in 189 health districts. ${ }^{3}$ It is clear from this audit and from reviewing the literature that there continue to be significant differences in clinical practice with respect to liver biopsy across the United Kingdom, and a lack of standardised protocols between institutions. These guidelines examine the evidence for methods of liver biopsy in adults.

\subsection{Formulation of guidelines}

2.1 VALIDITY AND GRADING OF RECOMMENDATIONS

The guidelines have been produced to conform with the North of England evidence-based guidelines development project. ${ }^{45}$

\subsubsection{Categories of evidence}

The strength of evidence used to formulate these guidelines was graded according to the following system:

Ia Evidence obtained from meta-analysis of randomised controlled trials.

Ib Evidence obtained from at least one randomised controlled trial.

IIa Evidence obtained from at least one well designed controlled study without randomisation.

$\mathrm{IIb}$ Evidence obtained from at least one other type of well designed, quasi-experimental study.

III Evidence obtained from well designed, non-experimental descriptive studies such as comparative studies, correlation studies and case studies.

Elizabeth Hospital, Birmingham B15 2TH, UK

A Grant

J Neuberger

Correspondence to: Professor Neuberger. Email: J.M.Neuberger@bham.ac.uk
The evidence category is indicated after the citations in the reference section at the end of these guidelines.

\subsubsection{Grading of recommendations}

The strength of each recommendation is dependent on the category of the evidence supporting it, and is graded according to the following system:

A Requires at least one randomised controlled trial as part of the body of literature of overall good quality and consistency addressing the specific recommendation (evidence categories Ia, Ib).

B Requires the availability of clinical studies without randomisation on the topic of recommendation (evidence categories IIa, IIb, III).

C Requires evidence from expert committee reports or opinions or clinical experience of respected authorities, in the absence of directly applicable clinical studies of good quality (evidence category IV).

\subsection{SCHEDULED REVIEW OF GUIDELINES}

As methods of diagnosis and tissue sampling change, new evidence will come to light and the content and evidence base for these guidelines should be reviewed frequently.

\subsection{Types of liver biopsy}

3.1 PERCUTANEOUS LIVER BIOPSY

Percutaneous liver biopsy may be classified according to the site of entry of the biopsy needle, whether the biopsy is performed in a blind or guided manner, or whether the biopsy track is plugged after the procedure.

\subsubsection{Transthoracic (transpleural or transparietal)} and subcostal liver biopsy

The patient lies supine for both of these approaches. The borders of the liver are usually defined by percussion or visualised by ultrasound. In most instances the intercostal space in the mid-axillary line just cephalad to the costal margin is then infiltrated with local anaesthetic, and a small incision is made through the dermis. The biopsy needle is then advanced into the intercostal space. The patient then holds his/her breath in expiration. The subsequent procedure for taking the biopsy then varies according to whether the biopsy needle is of the aspiration or cutting type.

Abbreviations used in these guidelines: CT, computed tomography; MRI, magnetic resonance imaging; PBC, primary biliary cirrhosis; PSC, primary sclerosing cholangitis; ERCP, endoscopic retrograde cholangiopancreatography; GGT, $\gamma$-glutamyl transpeptidase; BT, bleeding time; INR, international normalised ratio; BSG, British Society of Gastroenterology; FFP, fresh frozen plasma; AMA, antimitochondrial antibody. 
If the patient has an enlarged liver extending below the costal margin, then the site of entry of the biopsy needle may be subcostal. Complications are slightly more frequent with the transthoracic $(4.1 \%)$ than the subcostal route $(2.7 \%){ }^{6}$

After the biopsy procedure, the patient then lies on his/her right side or supine and pulse and blood pressure are monitored regularly in order to detect complications early (see section $7.10)$.

\subsubsection{Blind and guided liver biopsies}

A blind liver biopsy is one which is done as described earlier without imaging of the liver immediately prior to taking the biopsy sample.

A guided biopsy can be defined as a liver biopsy that is undertaken during real time imaging of the liver, whether that imaging modality be ultrasound, computed tomography (CT) or magnetic resonance imaging (MRI). Thus, guided biopsies should give access to thicker hepatic parenchyma, should avoid the puncture of adjacent organs, and should allow the accurate biopsy of focal hepatic lesions where appropriate. The use and evidence for image guided liver biopsy is controversial and will be discussed.

\subsubsection{Plugged liver biopsy}

Plugged liver biopsy is a modification of the percutaneous approach which was first described in $1984 .{ }^{78}$ It has been advocated as an alternative method for obtaining liver tissue in patients with impaired coagulation where transjugular biopsy is not available.

In this technique a biopsy samples is taken using a Tru-cut needle in the conventional manner (see section 3.1.2) but only the obturator containing the specimen is removed leaving the outer cutting sheath within the liver substance. A plastic cannula is then inserted down the sheath and while the breath is still held in expiration, gelatin or gel foam is injected as the sheath is withdrawn.

3.2 TRANSVENOUS (TRANSJUGULAR) LIVER BIOPSY Disorders of coagulation occur commonly in patients with liver disease and conventional practice in circumstances where there is significant disturbance of clotting is to avoid percutaneous liver biopsy because of the risk of bleeding, although the magnitude of this risk has not been defined in comparative studies.

Transvenous liver biopsy was first described in $1964^{9}$; this is usually done through a transjugular approach but may rarely be done via a transfemoral route. It is performed in a vascular catheterisation laboratory with videofluoroscopy equipment and cardiac monitoring because of the risk of cardiac arrhythmia as the catheter passes through the right atrium. The internal jugular vein is (usually) cannulated on the right side and a sheath inserted via a Seldinger technique. A $45 \mathrm{~cm}$ long catheter is then guided under fluoroscopic control through the right side of the heart to the inferior vena cava. The catheter is then loaded with the transvenous biopsy needle and advanced into the hepatic veins and the position checked by injection of contrast medium. The needle is then advanced rapidly $1-2 \mathrm{~cm}$ past the tip of the catheter with the patient holding his/her breath and the liver tissue is retained in the needle by aspiration on a syringe attached to the other end of the needle while it is inside the liver.

3.3 LAPAROSCOPIC LIVER BIOPSY

This technique is well established and its use varies widely between centres. In the United Kingdom it is often used for biopsying lesions found fortuitously at routine laparoscopic surgery. It has also been used in centres where access to transvenous liver biopsy is not available, for patients with abnormal clotting parameters, and also in patients who have a combination of a focal liver lesion and a coagulopathy where a histological diagnosis is essential in the management of that patient. Some centres in the USA perform laparoscopic liver biopsy on an outpatient basis ${ }^{10}$ and in some Japanese centres more than $50 \%$ of liver biopsies are performed laparoscopically. ${ }^{11}$

The complications in laparoscopic liver biopsy include those of the laparoscopy itself.

\subsection{Background}

The indications for, and methods of liver biopsy have changed over the past few years ${ }^{12}$ with the advent of new imaging techniques and the development of new indications for biopsy such as liver transplantation. ${ }^{13}$ All invasive procedures have a mortality rate associated with them, and consequently the benefits of obtaining liver for histology should always be weighed against the possible morbidity and mortality of the procedure.

\subsection{MORTALITY}

The reported mortality from percutaneous liver biopsy varies considerably. This is partly because most of the larger series reporting liver biopsy complications have been retrospective. ${ }^{14} 15$

The overall mortality rate in the three months after liver biopsy has been reported to be as high as $19 \% .^{3}$ Most of these deaths are the result of hepatic malignancy and advanced liver failure, and very few are due solely to the liver biopsy. The overall mortality rate also varies according to the centre in which the liver biopsies were performed - for example, in the Mayo Clinic the mortality from fatal haemorrhage after percutaneous biopsy was $0.11 \%,{ }^{16}$ whereas in an audit of liver biopsies performed in United Kingdom district general hospitals the death rate was between 0.13 and $0.33 \%$. $^{3}$

A generally accepted mortality rate in standard textbooks is between 0.1 and $0.01 \%{ }^{2}$

\subsubsection{Causes of mortality}

The main cause of mortality after percutaneous liver biopsy is intraperitoneal haemorrhage as shown in a retrospective Italian study of 68000 percutaneous liver biopsies in which all six patients who died did so from intraperitoneal haemorrhage. ${ }^{14}$ Three of these patients had had a laparotomy, and all had either cirrhosis or malignant disease, both of which 
are risk factors for bleeding. ${ }^{16}{ }^{17}$ Other serious complications responded to treatment; puncture of viscera was never followed by serious clinical complications. Other series have shown, however, that puncture of the gall bladder followed by biliary peritonitis is a recognised cause of death. ${ }^{3}$

As the main source of mortality after percutaneous liver biopsy is haemorrhage, it is reasonable to assume that improvements in mortality rates can be made if the clinician understands the risk factors for bleeding, recognises bleeding promptly and aggressively resuscitates the patient. It has been suggested that patients with suspected biliary peritonitis should have an early laparotomy. It has also been suggested that patients who bleed significantly (i.e. patients whose haemoglobin falls to $>20 \mathrm{~g} / 1$ or who become haemodynamically unstable) should be considered for either laparotomy or therapeutic angiography if the bleeding does not stop with transfusion alone. ${ }^{3}$

\subsection{MORBIDITY}

The overall morbidity from percutaneous liver biopsy is difficult to ascertain as most studies are retrospective and therefore symptoms such as post-biopsy pain requiring simple analgesia are not recorded. Although many groups have studied complications, there is no consensus about the division into major and minor symptoms and whether complications such as asymptomatic post-biopsy intrahepatic haematoma should be included in the figures. A morbidity rate of $5.9 \%$ for patients suffering minor complications after liver biopsy has been reported.

Pain is probably the commonest complication of liver biopsy occurring in up to $30 \%^{3} 18$ with moderate and severe pain occurring in 3 and $1.5 \%$, respectively. ${ }^{6}$ Hypotension and vasovagal episodes are common accompaniments to pain, occurring in about $3 \%$ of liver biopsies, ${ }^{6}$ and vasovagal episodes occasionally require the administration of atropine.

Significant haemorrhage (indicated by a drop in haemoglobin of $>20 \mathrm{~g} / \mathrm{l}$ ) occurs in $0.35-0.5 \%$ of all procedures. ${ }^{16}{ }^{19}$ Subclinical bleeding, however, occurs in a much higher percentage of patients, with up to $23 \%$ of patients having intrahepatic or subcapsular haematomas detectable by ultrasound 24 hours after biopsy. ${ }^{20}$ These haematomas are generally small and are not associated with significant haemodynamic compromise. Haemobilia occurs in $0.05 \%$ of patients and patients present with biliary pain, jaundice and melaena; arterial embolisation may rarely be required.

Puncture of other viscera occurs infrequently, with an incidence of between 0.01 and $0.1 \% .{ }^{14}$ The puncture of lung, colon, kidney and gall bladder together with pneumothorax, pleural effusion, and subcutaneous emphysema are well recognised complications, which rarely require intervention. ${ }^{21}$

Other recognised complications include sepsis, reaction to the anaesthetic, breakage of the biopsy needle, ${ }^{22}$ and intrahepatic arteriovenous fistulae. ${ }^{23}$
For other approaches, Riley and colleagues ${ }^{7}$ reported one case of a fatal haemorrhage after a plugged liver biopsy in a series of 20 patients. Lebrec and colleagues, ${ }^{15}$ in an analysis of 1000 transvenous liver biopsies, reported one death resulting from perforation of the liver capsule, and perforation of the liver capsule in five, haematoma at the site of cannulation in 10 , pneumothorax in two, transient supraventricular tachycardia in six, and abdominal pain in 74 patients.

\subsection{Indications for liver biopsy}

Percutaneous liver biopsy has a small but inherent risk even in the most experienced hands, and it should therefore only be performed when the benefits of knowing the histology outweigh the risks to the patient (in terms of altering treatment or defining disease outcome). These benefits should be continually re-evaluated as new treatment options become available such as has occurred with the new antiviral therapies in viral hepatitis and in liver transplantation.

Acute hepatitis of unknown aetiology, including possible drug related hepatitis, has long been an indication for percutaneous liver biopsy, but liver biopsy in typical acute viral hepatitis is usually not necessary. The usefulness of liver biopsy in chronic viral hepatitis was once hotly debated; however, with the advent of new antiviral therapies there is no doubt of the value of histology in assessing those patients who will benefit from treatment and assessing their response to it.

Patients with chronic hepatitis $\mathrm{C}$ virus infection as determined by a positive serum polymerase chain reaction test, who are being considered for antiviral therapy should undergo liver biopsy. Liver biopsy should probably be undertaken even if the patient has normal aminotransferases as it has been reported that up to $50 \%$ of patients with active disease have a normal serum alanine aminotransferase. ${ }^{24} \mathrm{~A}$ liver biopsy sample is useful in this instance in allowing an assessment of the Hepatitis Activity Index (a necroinflammatory/ fibrosis scoring system ${ }^{25}$ ) and to identify confounding factors such as alcoholic liver disease and haemochromatosis. Unfortunately, histology of a single liver biopsy sample and the monitoring of aminotransferases are poor predictors of disease progression. Consequently, repeat samples taken every two or three years may be needed to assess disease progression and prognosis.

In patients with raised serum ferritin or where disorders of copper metabolism are suspected, liver biopsy provides material for measurement of iron and copper within the liver parenchyma, although genetic analysis may help to differentiate genetic haemochromatosis from other causes of iron overload. Culture of biopsy material can help in the diagnosis of infections such as tuberculosis.

The need for liver biopsy in patients with intrahepatic cholestasis from primary biliary cirrhosis (PBC) and primary sclerosing cholangitis (PSC) is more controversial. On the one hand, the discovery that a persistently 
raised $\mathrm{E}_{2}$-antimitochondrial antibody (AMA) confirms a diagnosis of PBC (even if patients have no other signs or symptoms of PBC) means that a liver biopsy in the early stages of typical PBC (i.e., a middle aged woman with cholestasis) may be unnecessary. ${ }^{26}$ On the other, for more advanced disease liver biopsy may be useful in accurately staging the disease. The diagnosis of PSC related cholestasis is usually made at endoscopic retrograde cholangiopancreatography (ERCP) or MRI cholangiography, and diagnostic histological features in needle biopsy specimens are often not seen.

Liver biopsy is often useful in the diagnosis and management of patients with alcohol related liver diseases, as well as helping in the diagnosis of infections such as tuberculosis. Liver biopsy still remains part of the investigation of pyrexia of unknown origin and is also useful in the diagnosis of storage disorders.

Liver biopsy is often used in the investigation of abnormal liver enzymes but this must be taken in context, tempered by the results of other routine investigations, and take into account the patient's details. For example, the investigation of an isolated raised alkaline phosphatase will be very different in an 80 year old compared with a 25 year old. Raised $\gamma$-glutamyl transpeptidase (GGT) activities have been shown to be a sensitive marker of alcohol misuse; however, an isolated increase in GGT is not associated with major liver pathology and is therefore not an adequate indication on its own for liver biopsy. ${ }^{27}$

The role of percutaneous liver biopsy in the diagnosis of focal liver lesions depends largely upon the clinical picture. In most patients with malignant hepatocellular carcinoma ultrasound scanning, CT, and measurement of serum $\alpha$-fetoprotein will allow a diagnosis to be made (in the context of a space-occupying lesion in a cirrhotic patient). Similarly, a patient with a history of colonic resection for neoplasia who presents with a solitary lesion in the liver associated with raised serum carcinoembryonic antigen, may not require a biopsy of the lesion to make the diagnosis of a potentially resectable metastasis. Liver biopsy also carries a documented risk of seeding tumours down the biopsy track. ${ }^{28}$ The magnitude of this risk is currently unknown. Modern imaging techniques can also help to define other types of focal hepatic lesions such as haemangiomata and focal nodular hyperplasia. In these situations, some experts believe that the risk of bleeding after biopsy of a malignant tumour is greatest when the tumour is superficial and so recommend traversing normal liver before sampling tumour tissue. Fine needle aspiration biopsy may be a safer option if material for histological examination is required in the case of a suspected angioma. ${ }^{29}$

The use of liver biopsy after liver transplantation is increasing, and policies on histological monitoring vary between liver transplant units. Some units perform routine biopsies on day 7 after transplant to assess acute rejection, whereas others do annual review biopsies at which abnormalities are frequently seen. ${ }^{30}$ Liver biopsy is also useful in the diagnosis of invasive cytomegalovirus infection and in assessing recurrent disease. ${ }^{3132}$

Using liver biopsy in the context of research is controversial but has undoubtedly given invaluable information in the past in such areas as hepatitis C disease progression and the development of new drugs. We feel that these biopsies should be performed in the context of a clinical trial and where approval has been given by the local research ethics committee. In circumstances where the patient will derive no potential benefit from the procedure, and will thus only accrue the risks of that procedure, the patient should be fully aware of this and give written consent.

\subsection{Contraindications}

Many of the contraindications to percutaneous liver biopsy were defined by studies performed during the early years when liver biopsy was far less widely used than it is now. These studies were done before the advent of the Menghini "one second" technique and with larger diameter needles and although some of these contraindications seem to be common sense, many of them have been quoted as dogma in medical texts with very little evidence to support them.

6.1 THE UNCOOPERATIVE PATIENT

In percutaneous liver biopsy it is essential that the patient is cooperative as an untoward movement when the biopsy needle is in the hepatic parenchyma can lead to a tear of the liver and capsule and subsequent torrential bleeding. If the patient is frightened, then the use of midazolam as sedation can be considered with no increased risk. ${ }^{33}$ If the patient remains uncooperative and the benefit of obtaining liver histology outweighs the risk to the patient, then liver biopsy under general anaesthesia should be considered.

6.2 EXTRAHEPATIC BILIARY OBSTRUCTION Extrahepatic biliary obstruction is frequently quoted as a contraindication to liver biopsy which may be complicated by pain, biliary peritonitis, septicaemic shock, and death. ${ }^{34}$ However, in one study, serious complications in at least $2 \%$ of patients (including biliary peritonitis) and significant complications in another $4 \%$ followed the percutaneous liver biopsy. ${ }^{35}$ With current imaging techniques (specifically ERCP and MRI cholangiography), liver biopsies should only be performed in the context of biliary obstruction when there is doubt about the diagnosis and the benefit to the patient outweighs the risk. Under these circumstances the transjugular approach would be preferable. ${ }^{36}$

\subsection{BACTERIAL CHOLANGITIS}

The risk of inducing peritonitis and septic shock after liver biopsy has made cholangitis a relative contraindication. However, if a liver biopsy is performed when the biliary system is infected, then culture of a piece of liver can give useful bacteriological information especially in the context of investigation of tuberculosis or a pyrexia of unknown origin. Bacteraemia after 
percutaneous biopsy of a normal liver is a well recognised phenomenon ${ }^{37}$ and occurs in up to $14 \%$ of biopsies. ${ }^{38}$ These findings confirm the risks of disseminating infection at the time of liver biopsy.

\subsection{ABNORMAL COAGULATION INDEXES}

There are widely divergent opinions about the values at which abnormal coagulation indexes become contraindications to percutaneous liver biopsy. A number of investigators have shown that the degree of bleeding from the liver puncture site (observed at laparoscopy) bears no correlation to peripheral blood coagulation parameters, mentioned later, when these parameters are modestly increased. ${ }^{39} 40$ Some of these investigators have postulated that this discrepancy in liver bleeding time may be due to the inherent elasticity of the biopsy track collapsing down after the core has been taken, together with the high local concentrations of clotting factors within the hepatic parenchyma ${ }^{17}$ It should, however, be borne in mind that during a blind percutaneous liver biopsy, the liver is not the only structure to be punctured and the skin and subcutaneous tissues (and occasionally other organs) can bleed. Thus, peripheral indexes of clotting must still be taken into consideration.

Liver biopsy may be helpful in determining the extent of liver damage in patients with haemophilia and the benefits of treatment in those infected with hepatitis $C$ virus. In the absence of factor concentrate inhibitors, liver biopsy is safe if the clotting abnormalities are corrected before and for 24 hours after biopsy. ${ }^{42}$

\subsubsection{Prothrombin time}

Several large studies have failed to show an increased risk of bleeding associated with a prolongation of the prothrombin time of four seconds above control values. ${ }^{16} 17{ }^{39}$ The largest retrospective study of percutaneous liver biopsy to date failed to show any correlation between a prolongation of prothrombin time by seven seconds over control values and the occurrence of haemorrhagic complications. ${ }^{14}$ By contrast, a number of other studies, however, have corroborated the widely held belief that a coagulopathy predisposes the patient to haemorrhage after percutaneous liver biopsy. ${ }^{43}$ The 1991 BSG audit of the biopsy practice in 189 health districts in the United Kingdom showed that bleeding was commoner if the international normalised ration (INR) was raised, with $3.3 \%$ of the bleeds occurring when the INR was between 1.3 and 1.5 , and $7.1 \%$ occurring when the INR was $>1.5 .^{3}$ This suggests that about $90 \%$ of the bleeds occurred in patients with an INR $<1.3$ and reinforces the fact that having a normal INR or prothrombin time is no reassurance that the patient will not bleed after the procedure.

\subsubsection{Thrombocytopaenia}

The level at which thrombocytopaenia becomes a contraindication to percutaneous liver biopsy is uncertain from published data. One authority $^{44}$ proposes a platelet count above
$100000 / \mathrm{mm}^{3}$, whereas other groups such as the Mayo Clinic regard counts as low as $56000 / \mathrm{mm}^{3}$ to be safe. ${ }^{16}$ Most recognised UK texts require that the platelet count be above $80000 / \mathrm{mm}^{3},{ }^{2}$ whereas a survey of mostly US centres showed a preference for platelet counts above $50000 / \mathrm{mm}^{3} .{ }^{11}$ One study of 87 patients found that those patients with a platelet count below $60000 / \mathrm{mm}^{3}$ were significantly more likely to bleed after percutaneous liver biopsy than those with platelet counts above this value. ${ }^{45}$ The evidence for a cut off value remains scanty and takes no account of the function of the platelets (see section 6.4.3).

The effect on bleeding of thrombocytopaenia due to hypersplenism compared with thrombocytopaenia resulting from bone marrow failure has, to our knowledge, not been studied in detail.

The absolute value of the platelet count may not be crucial in determining the risk of bleeding as it is well recognised that even those patients with normal prothrombin times and platelet counts can have severely deranged bleeding times. Nevertheless, for a percutaneous liver biopsy the minimum platelet count felt to be safe without the need for support is $60000 / \mathrm{mm}^{3}$.

\subsubsection{Platelet function/bleeding time}

The practice of measuring bleeding time (BT) before liver biopsy is much more common in Asia compared with the USA (73 v 36\%). ${ }^{11}$ Our experience suggests that BT is seldom if ever measured in UK centres prior to liver biopsy even though the ingestion of aspirin and other non-steroidal anti-inflammatory drugs in the week prior to invasive intervention is a recognised contraindication by several authorities. There are to our knowledge, however, no convincing data to support this as a contraindication to percutaneous liver biopsy.

Patients with renal impairment usually have abnormalities of platelet function. According to one small study, patients with end stage renal failure on haemodialysis are at high risk (up to $50 \%$ ) of haemorrhagic complications after percutaneous liver biopsy, independent of the BT. ${ }^{46}$ This same study suggested that liver transplant recipients with a BT above $10 \mathrm{~min}$ utes (upper limit of normal) had a higher incidence of bleeding complications compared with those with a BT below 10 minutes. The sample size, however, is too small to allow any firm conclusions to be drawn.

Several other factors are likely to affect platelet function with or without affecting the BT. This fact, together with the considerable variation in results obtained between different operators, makes the use of BT as a measure of risk for haemorrhage difficult to interpret. The Royal Free Hospital was able to show that within a group of cirrhotic patients, those with abnormal BTs ( $42 \%$ ) were more likely to have significantly lower platelet counts, longer prothrombin times and higher blood urea and serum bilirubin than those with normal BTs (58\%). They also demonstrated that the bilirubin concentration as well as the platelet count were independently correlated with the 
BT (although the correlation for the latter was weak, and the raised serum bilirubin may well be just a surrogate marker for the severity of liver disease). ${ }^{47}$

6.5 ASCITES

Percutaneous biopsy of the liver in the presence of tense ascites is considered a contraindication in many texts. The reasons for this vary from the high likelihood of not obtaining a biopsy specimen because of the distance between the abdominal wall and the liver to the risk of uncontrollable bleeding into the ascites. Although these reasons seem to be sensible, they are not substantiated in randomised, controlled clinical trials. There is evidence, however, to support the fact that CT or ultrasound guided liver biopsy in the presence of ascites does not affect the complication rate. ${ }^{48} 49$

Notwithstanding these studies, it seems logical that if a liver biopsy is clinically indicated in a patient with tense ascites then there are several alternatives, the most obvious being to perform a total paracentesis prior to performing the percutaneous biopsy. Other options include image guided biopsy, transjugular liver biopsy, or laparoscopic biopsy.

\subsection{CYSTIC LESIONS}

Modern imaging techniques can often identify benign cystic lesions of the liver, thereby eliminating the need for biopsy in many cases. Cystic lesions within the liver may communicate with several structures including the biliary tree and therefore pose a risk of biliary peritonitis after biopsy.

The cystic lesion quoted most often as a contraindication to percutaneous liver biopsy was the echinococcal cyst because of the risk of dissemination of the hydatid cysts throughout the abdomen, and the risk of anaphylaxis. Recent advances in the treatment of hydatid disease of the liver mean that this may no longer be so. ${ }^{50}$ Aspiration of hydatid cysts with 19-22 gauge needles under ultrasound guidance has been shown to be safe and can be used both diagnostically ${ }^{51}$ and therapeutically ${ }^{52}$ for the injection of hypertonic saline or $95 \%$ ethanol under albendazole cover.

6.7 AMYLOIDOSIS

The use of liver biopsy in the diagnosis of amyloid liver disease was first used in 1928 . Volwiler and Jones reported the first death from haemorrhage after amyloid liver biopsy. ${ }^{53}$ This episode together with further reports of haemorrhage after liver biopsy in patients with amyloid have lead to the inclusion of amyloid liver disease in the list of contraindications to percutaneous liver biopsy. ${ }^{53}$ No large controlled trials have been performed to date which show an increased risk of haemorrhage after liver biopsy in amyloid liver disease. However, in 1961 a small series of liver biopsies in amyloid liver disease was reported. One of 18 patients had an intraperitoneal bleed but this patient was treated conservatively. ${ }^{54}$ Stauffer and colleagues $^{54}$ decided that liver biopsy was a useful method in the establishment of the diagnosis of hepatic amyloid, and certainly in the context of the investigation of hepatomegaly of uncertain aetiology this seems reasonable. However, if a diagnosis of amyloidosis had already been made or is strongly suspected, then a specific indication for performing a percutaneous liver biopsy is needed rather than for performing a more benign procedure such as a rectal biopsy.

\subsection{The biopsy procedure}

7.1 INFORMED CONSENT

Informed consent should be obtained in writing prior to the biopsy procedure in accordance with individual hospital policies. Consent forms should contain the patient's native language wherever possible, and when this is not possible there should be access to a competent interpreter to ensure adequate understanding by the patient of both the risks and benefits of the procedure and the commands given to them during the biopsy.

\subsection{EXPERIENCE OF THE OPERATOR}

There are no good data to show that the grade of the person performing the percutaneous liver biopsy has any affect upon the complication rate after the biopsy. The only data available are that from the 1991 BSG audit showing that the frequency of complications was slightly higher if the operator had performed less than 20 biopsies (frequency of complications was $3.2 \%$ if operator had performed $<20$ biopsies compared with $1.1 \%$ if the operator had performed $>100$ biopsies). No difference in the complication rates between gastroenterologists and general physicians was seen. ${ }^{3}$ A radiologist or clinician who is experienced in venous cannulation usually performs transjugular biopsies.

We recommend that pre-registration house officers should not perform percutaneous liver biopsies except in the context of specialised units, and then only under close supervision.

\subsection{SEDATION}

Anxious patients should be given the opportunity to have midazolam sedation for the biopsy procedure. Sedation should be given in accordance with the BSG guidelines on the administration of sedation for endoscopy. Midazolam should be given with caution in the context of liver disease.

\subsection{HAEMATOLOGICAL INVESTIGATIONS}

All patients undergoing percutaneous liver biopsy should have blood grouped and serum saved, and in hospitals where facilities for cross matching are limited, patients should have blood available.

The prothrombin time (or INR) and platelet count should be checked prior to the biopsy (preferably within 24 hours). With the current data it can be seen that there is no clear consensus as to the length of the prothrombin time at which the biopsy should not be performed. Consequently we feel that current advice should be followed and thus if the prothrombin time is prolonged by four seconds or more (or INR>1.4) then other strategies to 
improve the coagulopathy should be tried (see section 7.4.1).

The level of the platelet count at which a percutaneous liver biopsy should not be done is as controversial (see section 6.4.2); however, there is evidence that in patients with a platelet count as low as $60000 / \mathrm{mm}^{3}$, a percutaneous liver biopsy can be performed with no increase in complication rate.

\subsubsection{Vitamin K, fresh frozen plasma and platelet transfusion.}

Vitamin K, fresh frozen plasma (FFP) and platelet support are widely used for the correction of coagulation abnormalities prior to liver biopsy. There are, however, few data about the values at which correction of these coagulopathies should be abandoned in favour of plugged or transjugular biopsy. Vitamin $\mathrm{K}$ is useful but should be given parenterally and at least six hours before the biopsy, and is most effective where the disturbance in coagulation is caused by biliary obstruction or malabsorption. If this does not work then FFP given immediately prior to the biopsy in a dose of $12-15 \mathrm{ml} / \mathrm{kg}$ body weight may correct the prothrombin time. ${ }^{556}$ One study, however, has shown that FFP corrects the prothrombin time in only $20 \%$ of cases. ${ }^{57}$ Platelet transfusion prior to percutaneous liver biopsy in thrombocytopaenic patients has been used widely but has been hampered by the lack of studies showing its efficacy, especially in the context of patients with liver disease who may have other associated disorders of coagulation. It has been suggested that patients should initially receive 1 unit per $10 \mathrm{~kg}$ body weight and the effect of this transfusion be assessed by the platelet count obtained one hour later. ${ }^{58}$ However, posttransfusion platelet increments do not necessarily correlate with decreased risk of bleeding as platelet function may vary and it has been shown that $30 \%$ of patients receiving platelet transfusion show no improvement in in vitro bleeding time (a measure of platelet function). ${ }^{59}$

\subsection{PRE-BIOPSY ULTRASOUND}

Whether all patients about to undergo percutaneous liver biopsy should have an ultrasound is a contentious issue. Ultrasound is a safe and readily available investigation. Mainland European gastroenterologists are already required to be proficient in this method of imaging and it seems probable that before long all UK gastroenterologists will be trained to perform ultrasound at the bedside before or during the biopsy procedure. However, this is not current practice in the United Kingdom.

One of the reasons for performing a pre-biopsy ultrasound is to rule out anatomical variation-for example, Chilaiditi syndrome where bowel lies between the liver and the abdominal wall, thereby avoiding inadvertent puncture of an adjacent viscus. ${ }^{60}$ Ultrasound also permits the detection of focal lesions (which may or may not have been suspected) allowing for the opportunity of a targeted biopsy or fine needle aspiration at a later date under image guidance with a lower risk of haemorrhage.

Percussing for the superior and inferior borders of the liver is usually adequate for selection of the biopsy site ${ }^{61}$; however, in some patients where the borders of the liver are unclear (e.g. obese or cirrhotic patients) ultrasound is helpful.

\subsection{ULTRASOUND GUIDED PERCUTANEOUS LIVER} BIOPSY

Ultrasound guided percutaneous liver biopsy is used extensively in the investigation of focal liver lesions; however, its use in diffuse liver disease is more controversial. It has been postulated that ultrasound guided biopsy should reduce complications. As the commonest cause of mortality is bleeding, it follows that the incidence of bleeding should be proportional to the incidence of haematoma formation. The rate of haematoma formation however is unaffected by the use of ultrasound guidance. ${ }^{62}$ It is also difficult to understand why ultrasound should prevent haemorrhage (which is usually due to the rupture of large hepatic blood vessels) unless as postulated by Stotland and Lichtenstein, it leads to a reduction in the number of passes made into the liver. ${ }^{21}$ This may be especially important in the context of a shrunken liver where ultrasound may be used to perform the procedure accurately the first time. The increased risk of bleeding associated with multiple biopsy passes has been documented in patients with and without malignancy ${ }^{16}$ and has lead to the suggestion that all hepatic tumours should be biopsied by ultrasound or CT guided fine needle aspiration.

There is only one large series in which the use of ultrasound has been assessed in the context of diffuse liver disease. This paper was criticised for a number of reasons including the fact that it was retrospective and therefore subject to recall bias, that the sample size (although the largest study so far) was relatively small, and that the number of passes used in the control group was not documented (see section 8.8). ${ }^{21}$ This same paper quotes a significant reduction in major complications; however, there were no deaths and only one patient required therapeutic intervention in the form of a transfusion. ${ }^{63}$ These findings are consistent with a previous large retrospective study of 68276 biopsies, which concluded that complications of liver biopsy such as pneumothorax and puncture of other viscera seldom require intervention. $^{14}$

The use of ultrasound examination to assist in liver biopsy for non-focal disease has been estimated to be cost effective in the United States if the additional cost of ultrasound is less than US\$102 (£64). ${ }^{64}$

We believe that the use of guided liver biopsy or fine needle aspiration in the diagnosis of hepatic tumours is the safest way of managing these patients. It is also useful to have a prebiopsy ultrasound to rule out any anatomical abnormalities and in patients in whom the liver cannot be easily identified for reasons such as obesity. 
7.7 PROPHYLACTIC ANTIBIOTICS

Bacteraemia associated with liver biopsy in both structurally normal and abnormal livers has been well documented. ${ }^{37}{ }^{38}$ Therefore, prophylactic antibiotics should be used in the context of valvular heart disease or when there is previously documented bacteraemia.

Several groups have assessed the risks of septic complications for patients with choledochojejunostomy after liver transplantation. The conclusions of the Mayo group were that there was an increased risk $(12.5 \%)$ of septic complications in these patients, ${ }^{65}$ whereas the Royal Free group could show no increased risk providing there was no occult biliary obstruction. ${ }^{66}$ The latter study had too few patients to be able to make strong recommendations; however, other groups have come to the same conclusions. ${ }^{67}$

The current data on the use of prophylactic antibiotics are inconclusive and we feel that for patients in whom biliary sepsis is suspected it is prudent to use antibiotics.

7.8 TYPE OF BIOPSY NEEDLE

The two main types of needle currently being used in the United Kingdom are the Tru-cut and the Menghini needles. ${ }^{3}$ These two needles use different methods for sampling hepatic tissue. The former, as its name describes, is a cutting needle, whereas the latter uses a suction technique. These needles come in varying diameters, and the type and gauge of needle that is optimal for percutaneous liver biopsy have been the subject of several studies.

The largest series to look at needle type in relation to complications describes a complication rate of 3.5/1000 for the Tru-cut needle and $1 / 1000$ for the Menghini needle. Death, serious haemorrhagic complications, pneumothorax, and biliary peritonitis all occurred more frequently with the Tru-cut needle than with the Menghini needle, whereas puncture of other viscera and sepsis were more frequent with the Menghini needle. ${ }^{14}$ Other groups have compared the older Jamshidi suction needle with the Tru-cut/Vim Silverman cutting needles and found no difference in complication rates. ${ }^{6}{ }^{16}$ The theoretical advantages of the Menghini suction technique were described in the original paper, ${ }^{68}$ the main advantage being that the needle is only in the liver parenchyma for a "second". This allows less time for the patient to move, thereby minimising the potential for tearing the capsule.

The gauge of the biopsy needle and its effect on post-biopsy bleeding has been investigated for the suction style needle. One group showed that larger needles produced more bleeding after liver biopsy in anaesthetised pigs. This was statistically significant when comparing $2.1 \mathrm{~mm}$ (14 gauge) with $1.6 \mathrm{~mm}$ (16 gauge) needles, and also when comparing $1.6 \mathrm{~mm}$ with $1.2 \mathrm{~mm}$ (18 gauge) and smaller needles. ${ }^{40}$ Human studies of the effect of biopsy needle diameter on complications are rare; however, Forssell and colleagues ${ }^{18}$ could not show any difference in the incidence of intrahepatic haematoma formation when they compared the
$1.6 \mathrm{~mm}$ modified Menghini needle with a 1.9 mm Jamshidi needle.

The potential advantages of using smaller suction biopsy needles should be weighed against the disadvantages of having a smaller biopsy specimen. Specimens from the Tru-cut needles are larger and give more information about liver architecture and may thereby increase the diagnostic yield. The disadvantages of making several passes of the biopsy needle should also be borne in mind (see later).

7.9 NUMBER OF PASSES

It has been demonstrated that taking more than one core of liver at biopsy can increase the diagnostic yield, but this may have an effect on morbidity. It has been clearly shown that making more passes increases the incidence of complications when the percutaneous biopsy is taken by either transthoracic or subcostal approaches. In one paper the increased incidence reached significance when more than three biopsy samples were taken. ${ }^{6}$ This was subsequently confirmed by other studies showing that when blind percutaneous liver biopsy is undertaken, taking two specimens improves diagnostic yield with an increased number of minor complications when more than three consecutive specimens are taken. ${ }^{69}$

A large study of 9212 liver biopsies also showed that the risk of haemorrhage does not only increase with the number of passes made, but is also significantly linked to the age of the patient and the presence of malignancy. ${ }^{16}$ Therefore we conclude that under circumstances where the likelihood of a sampling error is high, such as in some cases of macronodular cirrhosis, two samples could be taken. However, the decision to do this for patients with advanced age or malignancy should be tempered by the increased risk of complications.

\subsection{POST-BIOPSY OBSERVATION}

The decision about the length of time that a patient should remain in hospital after a blind percutaneous liver biopsy is dependent on several factors. The main consideration in practical terms however is the likely time period in which complications are going to occur.

It has been shown that delayed haemorrhage can occur up to 15 days after percutaneous liver biopsy in patients who develop a postbiopsy coagulopathy. ${ }^{70}$ The occurrence of delayed haemorrhage is also documented after the reinstatement of warfarin therapy several days after percutaneous liver biopsy. Clearly, patients cannot be kept in hospital for two weeks or more after liver biopsy so a compromise has to be made on the basis of current knowledge.

The first large studies addressing the issue of post-biopsy observation were stimulated by the drive to perform outpatient percutaneous liver biopsies. These papers showed that the majority of complications occurred in the first three hours after liver biopsy, ${ }^{6}{ }^{19}$ and recommended that patients should be kept in hospital for six hours after the procedure. A later paper described $61 \%$ of complications after liver 
biopsy occurring in the first two hours, $82 \%$ of complications occurring in the first 10 hours, and $96 \%$ of complications occurring in the first 24 hours. In this paper recounting 68276 liver biopsies, six patients died, and all showed signs of bleeding within six hours of the procedure. ${ }^{14}$

The position that the patient should be nursed in after the liver biopsy has not been investigated, and various centres have differing policies including nursing the patient supine, on their right hand side or simply "flat". ${ }^{71}$ No controlled trials have been performed to assess these different techniques. Standard percutaneous liver biopsy observations include monitoring the patient's vital signs every 15 minutes for the first two hours, then every 30 minutes for two hours and then hourly for the rest of the remaining period. This protocol is reasonable when one considers that $61 \%$ of complications occur within the first two hours.

8.0 Outpatient percutaneous liver biopsy Outpatient percutaneous liver biopsy has been performed in many US centres since the early 1970s. ${ }^{6}$ In 1991 this practice had not been widely taken up in the United Kingdom with only $4 \%$ of percutaneous liver biopsies being performed as day cases. ${ }^{3}$ In centres which do perform day case biopsies in this country a $91 \%$ patient satisfaction rate has been quoted, and in carefully selected populations the admission rate to hospital after day case liver biopsy is $2.2-3.2 \% .^{71}$

In 1989 the American Gastroenterological Association published a consensus statement on outpatient percutaneous liver biopsy which we feel largely applies to UK patients. ${ }^{73}$ They recommended that patients undergoing this procedure should have no conditions that might increase the risk of the biopsy including: encephalopathy, ascites, hepatic failure with severe jaundice or evidence of significant extrahepatic biliary obstruction, significant coagulopathies or serious diseases involving other organs such as severe congestive heart failure or advanced age. We would add that patients with a strong suspicion of malignancy should not be biopsied as an outpatient because they have a 6-10 times higher risk of haemorrhage compared with patients without cancer. ${ }^{16}$

The consensus statement also recommends that the place where the biopsy is performed should have easy access to a laboratory, blood bank and inpatient facilities should the need arise, and there should be staff to observe the patients for six hours. The patient should be admitted to hospital if there is any significant complication including pain requiring more than one dose of analgesic in the four hours after liver biopsy. The patient should also be able to return easily to the hospital where the biopsy was undertaken within 30 minutes, and should have a reliable individual to stay with on the first post-biopsy night.

If the above criteria cannot be met, then the patient should not be biopsied as an outpatient.

Performing percutaneous liver biopsies as an outpatient has considerable potential for cost saving and reallocation of resources. ${ }^{6}$

\section{Recommendations}

- Before performing a percutaneous liver biopsy, there must be a clearly defined indication for the biopsy, and the risks to the patient should not outweigh the potential benefits.

- We recommend that all patients who are about to undergo a percutaneous liver biopsy should have had some form of imaging of the liver within the preceding four weeks. This will allow the detection of abnormal anatomy in the area of the proposed biopsy (see section 7.5 ), while at the same time detecting focal lesions which should be biopsied under image guidance. Recommendation grade $B$.

- The patient's platelet count and prothrombin time should be checked in the week before the percutaneous liver biopsy providing that the patient's liver disease is stable.

- If the platelet count is $>60000 / \mathrm{mm}^{3}$ then the biopsy can be safely performed. If the platelet count is $40000-60000 / \mathrm{mm}^{3}$ then platelet transfusion may increase the count enough for the biopsy to be performed safely by the percutaneous route. If, however, platelet transfusion does not increase or the platelet count is $<40000 / \mathrm{mm}^{3}$ then alternative biopsy methods such as plugged, transvenous (transjugular), or laparoscopic liver biopsy can be tried (see sections 6.4 and 7.3). Recommendation grade $B$.

- If the prothrombin time is $<4$ seconds prolonged, then percutaneous biopsy can be safely undertaken. If the prothrombin time is $4-6$ seconds prolonged then, a transfusion of fresh frozen plasma may bring the prothrombin time into the desired range (see sections 6.4 and 7.4 ). If the prothrombin time is $>6$ seconds prolonged then other biopsy methods should be tried. Recommendation grade $B$.

- Informed consent should be obtained from all patients prior to percutaneous liver biopsy in accordance with local hospital guidelines. The patient should also be able to understand and cooperate with instructions given by the person performing the liver biopsy (see section 7.1).

- Sedation with midazolam may be given for percutaneous liver biopsy in accordance with the BSG guidelines on sedation during endoscopy. Sedation should be given with caution in liver disease (see section 6.1). Recommendation grade $B$.

- The type of needle used for the biopsy will depend on the experience of the operator and the type of needle they are used to. Where a larger biopsy is not required the Menghini needle should be used in preference to cutting needles as this technique seems to have a lower complication rate (which may however be at the expense of the diagnostic yield). Where the operator has only experience of one style of needle they should use the technique most familiar to them (see section 7.8). Recommendation grade $A$. 
- The grade of the operator has not been shown adversely to affect the complication rate from percutaneous liver biopsy. We feel, however, that doctors who have performed less than 20 biopsies should not perform the procedure unsupervised and that house officers should not be performing percutaneous liver biopsies except in the context of a busy specialised gastrointestinal unit (see section 7.2). Recommendation grade $B$.

- Prophylactic antibiotics should be given to patients with valvular heart disease or those at risk of bacteraemia (section 7.7). Recommendation grade $B$.

- Usually one pass of the biopsy needle retrieves enough hepatic tissue for diagnostic purposes; however, if there may be a sampling error (such as may occur in macronodular cirrhosis) which will result in an inappropriate diagnosis, then two passes may be made without significantly affecting the complication rate (see section 7.9.). Recommendation grade $B$.

- We recommend that patients undergoing outpatient percutaneous biopsy should not have any condition that may increase the risk of the biopsy procedure (see section 8). Recommendation grade $B$.

- Post liver biopsy observation should continue for six hours and if at the end of this period there have been no complications then the patient may be discharged. The patient should, however, have a responsible person to stay with on the first post-biopsy night and should be able to return to hospital within 30 minutes should the need arise (see section 7.10). Recommendation grade $B$.

Dr A J Grant and Professor J Neuberger, University Hospital, Birmingham, UK, have formulated these guidelines. Within the boundaries of current literature we have attempted where possible to make the guidelines evidence-based.

\section{References}

1 Bingel A. Ueber die parenchympunktion der leber. Verh Dtsch Ges Inn Med 1923;35:210-12.

2 Sherlock S, Dooley J. Diseases of the liver and biliary system. 10th edn. London: Blackwell Scientific, 1997.

3 Gilmore IT, Grade: IV IT, Burroughs A, Murray-Lyon IM, et al. Indications, methods, and outcomes of percutaneous liver biopsy in England and Wales: an audit by the British Society of Gastroenterology and the Royal College of Physicians of London. Gut 1995;36:437-41.

Grade: III

4 Eccles M, Clapp Z, Grimshaw J, et al. North of England evidence based guidelines development project: methods of guidelines development. BMF 1996;312:760-2.

5 Grimshaw J, Eccles M, Russell I. Developing clinically valid practice guidelines. Fournal of Evaluation in Clinical Practice practice guideline

6 Perrault J, McGill DB, Ott BJ, et al. Liver biopsy: complications in 1000 inpatients and outpatients. Gastroenterology 1978;74:103-6.

7 Riley SA, Ellis WR, Irving HC, et at. Perc biopsy with plugg, Irving HC, et al. Percutaneous liver biopsy with plugging of needle track: a safe method for use in patients with impaired coagulation. Lancet 1984;ii:436.

8 Tobin MV, Gilmore IT. Plugged liver biopsy in patients with impaired coagulation. Dig Dis Sci 1989:34:13-15.

Grade: IIb

9 Dotter CT. Catheter biopsy. Experimental technique for transvenous liver biopsy. Radiology 1964;82:312-14.

Grade: IV

10 Lightdale CJ, Das L. Difficult liver biopsies: Only for radiologists? Am f Gastroenterol 1997;92:364-5.

1 Sue M, Caldwell SH, Dickson RC, et al. Variationade: IV centres in technique and guidelines for liver biopsy. Liver centres in technique

Grade: III
12 Lebrec D. Various approaches to obtaining liver tissuechoosing the biopsy technique. F Hepatol 1996;25(suppl 1):20-4.

13 Hübscher SG, Clements Grade: IV Hübscher SG, Clements D, Elias E, et al. Biopsy findings in cases of rejection of liver allograft. $\mathcal{F}$ Clin Pathol 1985;38: 1366-73.

Grade: III

14 Piccininio F, Sagnelli E, Pasquale G, et al. Complications following percutaneous liver biopsy. $\mathcal{F}$ Hepatol 1986;2:16573

15 Lebrec D, Goldfarb G, Degott C, et al. Transvenous liver biopsy. Gastroenterology 1982;83:338-40.

Grade: III

16 McGill DB, Rakela J, Zinsmeister AR, et al. A 21-year experience with major haemorrhage after percutaneous experience with major haemorrhage after percut
liver biopsy. Gastroenterology 1990;99:1396-400.

Grade: IIa

17 Ewe K. Bleeding after liver biopsy does not correlate with indices of peripheral coagulation. Dig Dis Sci 1981;26:388-

18 Forssell PL, Bronkowsky HL, Anderson PB, et al. Intrahepatic haematoma after aspiration liver biopsy: a prospective randomised controlled trial using two different needles. Dig Dis Sci 1981;26:631-5.

Grade: Ib

19 Knauer MC. Percutaneous biopsy of the liver as a procedure for outpatients. Gastroenterology 1978;74:101-2.

Grade: III

20 Minuk GY, Sutherland LR, Wiseman D, et al. Prospective study of the incidence of ultrasound-detected intrahepatic and subcapsular haematomas in patients randomized to 6 or 24 hours of bed rest after percutaneous liver biopsy. Gastroenterology 1987;92:290-3.

Grade: Ib

21 Stotland BR, Lichtenstein GR. Liver biopsy complications and routine ultrasound. Am F Gastroenterol 1996;91:1295-6. Grade: IV

22 Lazar H. Fractured liver biopsy needles. Gastroenterology 1978;74:801.

Grade: III

23 Okuda K, Musha H, Nakajima Y, et al. Frequency of intrahepatic arteriovenous fistula as a sequelae to percutaneous needle puncture of the liver. Gastroenterology 1978;74: $1204-7$.

Grade: IV

24 Alberti A, Morsica G, Chemello L, et al. Hepatitis C viraemia and liver disease in symptom-free individuals with anti-HCV. Lancet 1992;340:697-8.

25 Knodell RG, Conrad ME, Ishak KG. Development of chronic liver disease after acute non-A, non-B, post
transfusion hepatitis. Gastroenterology 1977;72:902-9.

26 Metcalf JV, Mitchison HC, Palmer JM, et al. Natural history of early primary biliary cirrhosis. Lancet 1996;348:1399402

Grade: IIb

27 Ireland A, Hartley L, Ryley $\mathrm{N}$, et al. Raised $\gamma$-glutamyltransferase activity and the need for liver biopsy. BMF 1991;302:388-9.

Grade: III

28 Hamazaki K, Matsubara N, Mori M, et al. Needle tract implantation of hepatocellular carcinoma after ultrasonically guided needle liver biopsy. F Hepatogastroenterol 1995; 42:601-6.

29 Caldironi MW, Gazzucco M, Aldinio $M$ G IV guided fine-needle biopsy for the diagnosis of hepatic angioma. Minerva Chir 1998;53:505-9.

Grade: III

30 Nakhleh RE, Schwartzenberg SJ, Bloomer J, et al. The pathology of liver allografts surviving longer than one year. Hepatology 1990;11:465-70.

Grade: III

31 Hübscher SG, Elias E, Buckels JAC, et al. Primary biliary cirrhosis. Histological evidence for disease recurrence after liver transplant. F Hepatol 1993;18:173-84.

Grade: III

32 Harrison RF, Davies M, Goldin RD, et al. Recurrent hepatitis B. A distinct form of rapidly developing cirrhosis. Histopathology 1993;20:112-16.

Grade: III

33 Alexander JA, Smith BJ. Midazolam sedation for percutaneous liver biopsy. Dig Dis Sci 1993;38:2209-11.

34 LoIudice T, Buhac I, Balint J. Septicaemia Grade: IIa of percutaneous liver biopsy. Gastroenterology 1977;72:949-

Grade: III

35 Morris JS, Gallo GA, Scheuer PJ, et al. Percutaneous liver biopsy in patients with large bile duct obstruction. Gastroenterology 1975;68:750-4.

Grade: III

36 Rosch J, Lakin PC, Antonovic R, et al. Transjugular approach to liver biopsy and transhepatic cholangiography. N Engl F Med 1973;289:227-31.

37 McCloskey RV, Gold M, Weser E. Bacteraemia after liver biopsy. Arch Intern Med 1973;132:213-15.

Grade: IIb

38 Le Frock JL, Ellis CA, Turchik JB, et al. Transient bacteraemia associated with percutaneous liver biopsy. F Infect Dis 1975;131:S104-7.

Grade: IIb 
39 Dillon JF, Simpson KJ, Hayes PC. Liver biopsy bleeding time-an unpredictable event. $\mathcal{f}$ Gastroenterol Hepatol time-an unpredi

Grade: IIb

40 Gazelle GS, Haaga JR, Rowland D. Effect of needle gauge, level of anticoagulation, and target organ on bleeding associated with aspiration biopsy. Radiology 1992;183:509-13.

41 Wong VS, Baglin T, Beacham E, et al. The role for liver biopsy in haemophiliacs infected with the hepatitis $C$ virus. Br f Haematol 1997;97:343-7.

Grade: IIb

42 Ahmed MM, Mutimer DJ, Elias E, et al. A combined management protocol for patients with coagulation disorders infected with hepatitis C virus. Br F Haematol 1996;95:3838.

Grade: IIb

43 Mahal AS, Knauer CM, Gregory PB. Bleeding after liver biopsy: how often and why? Gastroenterology 1979;76:1192.

Grade: III

44 Menghini G, Antonini R, Bruschelli P. Open abdomen liver biopsy by a modified one-second technic. Am $\mathcal{F}$ Surg 1977; 133:383

45 Sharma P, McDonald GB, Banaji M. The risk of bleeding after percutaneous liver biopsy: relation to platelet count. $\mathscr{F}$ Clin Gastroenterol 1982;4:451-3.

Grade: III

46 Wolf DC, Weber F, Palascak I, et al. Role of the template bleeding time in predicting bleeding complications of percutaneous liver biopsy [abstract]. Hepatology 1995;22: $509 \mathrm{~A}$

Grade: IIb

47 Blake JC, Sprengers D, Grech P, et al. Bleeding time in patients with hepatic cirrhosis. BMF 1990;301:12-15.

48 Little AF, Ferris JV, Dodd GD, et al. Image guidrade: II neous hepatic biopsy: Effect of ascites on the complication rate. Radiology 1996;199:79-83.

49 Murphy FB, Barefield KP, Steinberg HV, Grade: II sonography-guided biopsy of the liver in the presence of sonography-guided biopsy of the liver in the presence of
ascites: frequency of complications. AfR Am $\mathcal{F}$ Roentgenol ascites: frequency

50 Kumar A, Chattopadhyay TK, Grade: IIa ease of the liver. Postgrad Med f 1992;68:853-6. Bret PM, Fond A, Bretagnolle $M$, et al. Percutaneous ogy 1988;168:617-20.

Grade: IIb

52 Filice C, Pirola F, Brunetti E, et al. New therapeutic approach for hydatid liver cysts. Gastroenterology 1990;98: 1366-8.

Grade: III

53 Volwiler W, Jones CM. The diagnostic and therapeutic value of liver biopsies; with special reference to trocar biopsy. $N$ of liver biopsies; with spe
Engl f Med 1947;237:651.

54 Stauffer $\mathrm{MH}$, Gross JB, Foulk WT, et al. Amy Grade: III Stauffer MH, Gross JB, Foulk WT, et al. Amyloidosis: Diag-
nosis with needle biopsy of the liver in 18 patients. Gastronosis with needle biopsy
enterology 1961;41:92-6.

Grade: III

5 Spector MD, Corn M, Ticktin HE. Effect of plasma transfusions on the prothrombin time and clotting factors in liver disease. N Engl f Med 1966;275:1032-7.

56 Contreras M, Ala FA, Greaves M, et al. Guidelines for the use of fresh frozen plasma. Transfus Med 1992;2:57-63.

Grade: I
57 Gazzard BG, Henderson JM, Williams R. The use of fresh frozen plasma or a concentrate of factor IX as replacement therapy before liver biopsy. Gut 1975;16:621-5. 1987;257:1777-80.

Grade: IV

59 Kristensen J, Eriksson L, Olsson K, et al. Functional capacity of transfused platelets estimated by the Thrombostat 4000/2. Eur f Haematol 1993;51:152-5.

Grade: IIb neous liver biopsy: Anatomical variation. Lancet 1987;ii: 437-9.

Grade: III

61 Qureshi WA, DuBose TJ. Effect of operator experience on liver biopsy site selection [abstract]. Gastroenterology 1997; 112:A37. Vautier G, Scott B, Jenkins D. Liver biopsy: blind or guided?
$B M \mathcal{F}$ 1994;309:1455-6.

Grade: IV

63 Caturelli E, Giacobbe A, Facciorusio D, et al. Percutaneous biopsy in diffuse liver disease: Increasing diagnostic yield and decreasing complication rate by routine ultrasound assessment of puncture site. Am f Gastroenterol 1996;91: 1318-21. Grade: IIa
UltrasoundYounnossi ZM, Teran JC, Ganiats TG, et al. Ultrasound-
guided liver biopsy for parenchymal liver disease: an economic analysis. Dig Dis Sci 1998;43:46-50.

65 Bubak ME, Porayko MK, Krom RAF, et al. Complications of liver biopsy in liver transplant patients: increased sepsis associated with choledochojejunostomy. Hepatology 1991; 14:1603-5.

Grade: III

66 Ben-Ari Z, Neville L, Rolles K, et al. Liver biopsy in liver transplantation: no additional risk of infections in patients with choledochojejunostomy. F Hepatol 1996;24:324-7.

Grade: IIa

67 Galati JS, Monsour HP, Donovan JP, et al. The nature of complications following liver biopsy in transplant patients with Roux-en-Y choledochojejunostomy. Hepatology 1994; 20:651-3. Menghini G. One-second biopsy of the liver-problems of its clinical application. $N$ Engl f Med 1970;283:582-5.

69 Maharaj B, Bhoora IG. Complications associated with percutaneous needle biopsy of the liver when one, two, or three specimens are taken. Postgrad Med f 1992;68:964-7.

Grade: III rhage after percutaneous liver biopsy. $f$ Clin Gastroenterol 1983;5:263-6

Grade: IV

71 Douds AC, Joseph AEA, Finlayson C, et al. Is day case liver biopsy underutilised? Gut 1995;37:574-5.

Grade: III

72 Janes $\mathrm{CH}$, Lindor KD. Outcome of patients hospitalised for complications after outpatient liver biopsy. Ann Intern Med 1993;118:96-8.

Grade: III

73 Jacobs WH, Goldberg SB. Statement on out-patient percutaneous liver biopsy. Dig Dis Sci 1989;34:322-3.

Grade: IV 